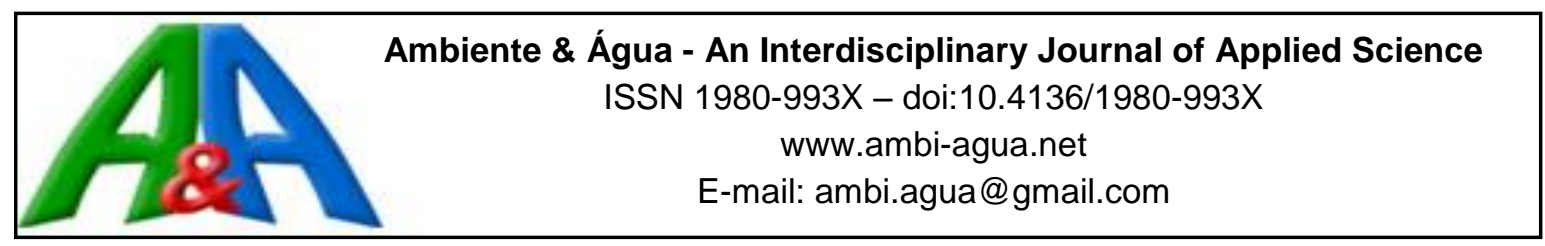

\title{
Ocorrência e remoção de estrogênios por processos de tratamento biológico de esgotos
}

\author{
doi:10.4136/ambi-agua.1992
}

Received: 24 Aug. 2016; Accepted: 20 Dec. 2016

\author{
Danieli Lima da Cunha ${ }^{1,2 *}$; Lícia Murito de Paula ${ }^{1}$; \\ Samuel Muylaert Camargo da Silva ${ }^{3}$; Daniele Maia Bila ${ }^{4}$; \\ Estefan Monteiro da Fonseca ${ }^{2}$; Jaime Lopes da Mota Oliveira ${ }^{1}$ \\ ${ }^{1}$ Fundação Oswaldo Cruz (FIOCRUZ), Rio de Janeiro, RJ, Brasil \\ Departamento de Saneamento e Saúde Ambiental \\ ${ }^{2}$ Universidade Federal Fluminense (UFF), Niterói, RJ, Brasil \\ Departamento de Geologia \\ ${ }^{3}$ Universidade do Estado do Rio de Janeiro (UERJ), Rio de Janeiro, RJ, Brasil \\ Programa de Pós-Graduação em Gestão e Regulação de Recursos Hídricos \\ ${ }^{4}$ Universidade do Estado do Rio de Janeiro (UERJ), Rio de Janeiro, RJ, Brasil \\ Departamento de Engenharia Sanitária e do Meio Ambiente \\ *Autor correspondente: e-mail: danielicunha@ hotmail.com, \\ liciamurito@gmail.com,samu_muylaert@hotmail.com, \\ danielebilauerj@gmail.com, oceano25@hotmail.com, \\ jaimelmoliveira@gmail.com
}

\section{RESUMO}

Os estrogênios são micropoluentes capazes de causar alterações no sistema endócrino de organismos aquáticos. Uma das principais fontes da sua introdução no meio hídrico é através do lançamento de esgotos domésticos. Este artigo apresenta uma revisão sobre a ocorrência dos estrogênios estrona, estradiol, estriol e etinilestradiol em esgotos brutos e tratados e em águas superficiais, bem como sobre sua remoção por diferentes processos de tratamento biológico de esgoto. Os poucos trabalhos realizados no Brasil mostram que a concentração desses compostos nestas matrizes é expressiva. Além disso, foi observado um decaimento nas concentrações desses estrogênios do esgoto bruto ao tratado. Os sistemas aeróbios, como lodos ativados, mostraram melhor desempenho na remoção de estrogênios, sobretudo por meio da sorção ao lodo. No entanto, pouco se sabe sobre os mecanismos de degradação de tais compostos nestes processos e sobre o comportamento de seus conjugados. Portanto, os processos de tratamento biológico de esgoto representam uma barreira à introdução de estrogênios nos corpos hídricos.

Palavras-chave: estrogênios em esgoto, remoção, tratamento biológico.

\section{Occurrence of estrogens and their removal by biological processes of sewage treatment}

\begin{abstract}
Estrogens are micropollutants that may harm aquatic organisms endocrine system. They enter water bodies mainly through domestic sewage. This paper aims to review the estrone, estradiol, estriol and ethinyl estradiol occurrence in untreated and treated sewage and surface water, as well as their removal efficiency through employment of different sewage biological
\end{abstract}


treatment methods. The few studies carried out in Brazil show a significant concentration of these compounds in the reviewed sources, with a downward trend from raw to treated sewage. Aerobic systems, such as activated sludge, showed better results at removing the estrogens, mainly through adsorption by sludge. However, little is known about the degradation mechanisms of such compounds and the behavior of their conjugates. Therefore, biological processes of sewage treatment represent a barrier to the introduction of estrogens to the water bodies.

Keywords: biological treatment, estrogens in the sewage, removal.

\section{INTRODUÇÃO}

Com o avanço da ciência e do conhecimento sobre os efeitos decorrentes da poluição dos corpos hídricos, alguns países da União Europeia e os Estados Unidos vêm buscando ampliar a regulamentação e o monitoramento de novos compostos, como os desreguladores endócrinos, que antes não eram objeto de atenção por parte dos dispositivos legais (Cunha et al., 2016).

Estudos mostram que essas substâncias, mesmo em concentrações extremamente baixas (ng $\mathrm{L}^{-1}$ ), podem promover alterações no sistema endócrino dos organismos aquáticos. Esses compostos são capazes de desencadear efeitos adversos como interferir no crescimento, desenvolvimento e/ou reprodução desses organismos (Barceló e Petrovic, 2008; Bila e Dezotti, 2007). Em face disto, a comunidade científica busca compreender melhor as vias de introdução e dispersão desses compostos no meio ambiente e os possíveis mecanismos de sua remoção. $\mathrm{O}$ maior entendimento da dinâmica destes compostos no meio ambiente permitirá o aperfeiçoamento das tecnologias de remoção através dos processos de tratamento de efluentes.

Dentre este grupo de substâncias, os estrogênios merecem especial atenção, uma vez que a sua presença em efluentes urbanos tem sido diretamente correlacionada a impactos à biota aquática. Estudos ecotoxicológicos apontam que a exposição contínua aos estrogênios está associada a alterações bioquímicas e histopatológicas, redução significativa na taxa de fertilização, diminuição na eclosão de ovos e modificações comportamentais no acasalamento em espécies de peixes, anfíbios, crustáceos e gastrópodes (Silva et al., 2012; Bergman et al., 2012; Giusti et al., 2014; Luna et al., 2015; Garmshausen et al., 2015). O efeito mais alarmante é o processo de feminização (desenvolvimento de características sexuais femininas em machos, incluindo anatomia reprodutiva feminina), uma vez que compromete o ciclo reprodutivo de toda uma população, podendo assim desencadear um desequilíbrio ecossistêmico (Gilbert, 2012).

Neste contexto de risco à biota aquática, o lançamento de esgoto "in natura" ou tratado tem sido indicado como principal contribuinte para a introdução de estrogênios no meio hídrico. Dessa forma, os processos de tratamento podem representar uma barreira para seu lançamento nos corpos hídricos. Porém, para que a depuração seja efetiva, é necessário implementar sistemas que possam realmente remover e/ou degradar tais compostos. No entanto, observa-se que os processos convencionais de tratamento de esgotos podem não remover integralmente esses compostos e, desta forma, conduzir a sua introdução contínua no meio hídrico (Brandt et al., 2013; Aquino et al., 2013).

Diante da crescente preocupação com a qualidade da água e a fim de minimizar os riscos causados pela poluição hídrica, países têm investido em estudos para o aprimoramento das tecnologias de tratamento de águas residuais. Portanto, este trabalho tem como objetivo apresentar uma revisão sobre a ocorrência dos estrogênios estrona, estriol, estradiol e etinilestradiol nas águas residuais e superficiais. Além disso, este artigo mostra a eficiência encontrada por alguns autores em relação à remoção desses compostos por processos biológicos para o tratamento de esgoto.

Rev. Ambient. Água vol. 12 n. 2 Taubaté - Mar. / Apr. 2017 


\section{CARACTERÍSTICAS FÍSICO-QUÍMICAS E METABÓLICAS}

Os estrogênios, bem como todos os hormônios esteroidais, possuem uma mesma estrutura química básica de 17 átomos de carbono dispostos em quatro anéis (A, B, C e D) ligados entre si. Configurações estruturais no anel $\mathrm{D}$ dão origem a diferentes estrogênios (Tabela 1). Na posição do carbono 17 (C17) a estrona possui uma carbonila enquanto o estradiol possui uma hidroxila, já o estriol possui duas hidroxilas, uma no C16 e a outra no C17.

O etinilestradiol difere-se do estradiol através de um único radical, o etinil, também no carbono 17. Esta diferença faz com que o etinilestradiol possua maior potencial estrogênico e também se torne mais resistente à degradação com relação ao estradiol (Combalbert e Hernandez-Raquet, 2010; WHO e IARC, 2007).

Tabela 1. Propriedades físico-químicas de alguns estrogênios (Hamid e Eskicioglu 2012).

Estrogênio

a CAS: Chemical Abstract Service. Número que corresponde à identificação química da substância.

b K ow: Coeficiente de partição octanol/água.

c $\mathrm{pK}_{\mathrm{a}}$ : Constante de dissociação ácida.

$\mathrm{d} \mathrm{K}_{\mathrm{d}}$ : Coeficiente de distribuição.

Com relação as propriedades físico-químicas, um parâmetro importante e frequentemente usado na descrição do comportamento de um contaminante no ambiente é o coeficiente de distribuição $\left(\mathrm{K}_{\mathrm{d}}\right)$. Este por sua vez está diretamente associado ao coeficiente de partição octanol/água $\left(\mathrm{K}_{\mathrm{ow}}\right)$.

$\mathrm{O} \mathrm{K}_{\mathrm{d}}$ é determinado pela razão entre a massa do composto na fase sólida e na fase líquida. Este parâmetro é muito útil para estimar o potencial de sorção do contaminante dissolvido em contato com o solo. Quanto maior o $\mathrm{K}_{\mathrm{d}}$, maior a tendência do contaminante ficar adsorvido ao solo ou sedimento (CETESB, 2001). 
Ainda com relação à mobilidade dos estrogênios, o $\mathrm{K}_{\mathrm{ow}}$, definido pela a relação entre concentração do contaminante orgânico na fase octanol e a concentração deste na fase aquosa, se apresenta como importante coeficiente para compreender a hidroafinidade destes compostos. Valores de $\log \mathrm{K}_{\text {ow }}$ para os estrogênios variam entre 2,45 e 4. Este resultado mostra que os mesmos são moderadamente hidrofóbicos, tendo a tendência de se associarem à fase sólida.

No que diz respeito às características metabólicas, a maioria dos estrogênios produzidos pelo corpo humano, ou mesmo aqueles que são ingeridos através de anticoncepcionais e/ou de medicamentos utilizados na terapia de reposição hormonal, são excretados pelas fezes e urina em sua forma conjugada (sulfato e/ou glicuronídeos) (Cano et al., 1997). Tais conjugados polares são biologicamente menos ativos e mais solúveis em água quando comparados aos estrogênios livres, não conjugados (D'ascenzo et al., 2003).

Vale observar que, mesmo com estes mecanismos de degradação, estudos constataram a ocorrência de estrogênios em sua forma livre em diferentes compartimentos ambientais, principalmente em afluentes e efluentes de ETE. Isso ocorre devido ao um processo natural, no qual as bactérias presentes no meio, principalmente a Escherichia coli, são capazes de desconjugar os estrogênios, tornando-os novamente a sua forma livre (Fent et al., 2006; D'ascenzo et al., 2003).

Este processo ocorre, pois, a $E$. coli é capaz de sintetizar uma grande quantidade da enzima $\beta$-glucuronisidase, responsável por essa transformação. A natureza recalcitrante dos conjugados sulfatos ao longo das ETEs pode ser explicada por uma atividade mais fraca da arilsulfatase da E. coli comparada com a $\beta$-galogsidade (Hamid e Eskicioglu 2012).

\section{OCORRÊNCIA DE ESTROGÊNIOS NO ESGOTO BRUTO, TRATADO E NA ÁGUA SUPERFICIAL}

Uma vez constatada a introdução contínua de estrogênios no meio hídrico e seus efeitos negativos à qualidade das águas e, consequentemente, à saúde dos ecossistemas, o monitoramento destas substâncias vem ganhando especial atenção. Assim, métodos têm sido desenvolvidos visando a determinação desses compostos nas mais variadas matrizes aquáticas.

A Figura 1 resume as concentrações de estrogênios relatadas por diversos estudos, que analisaram esgoto bruto, esgoto tratado e águas superficiais em diversos países, com exceção do Brasil.

Para construção do gráfico apresentado na Figura 1 foram consultados os dados obtidos de cerca de 15 artigos, conduzidos em diferentes países. Estes foram então introduzidos e processados no programa estatístico SPSS versão 22.0, optando-se por gerar um gráfico em formato boxplot. Na Figura 1, são apresentadas as barras principais, limitadas pelo primeiro e terceiro quartil; as medianas; e as hastes dos limites inferior e superior. Nesta representação, foram excluídos os pontos discrepantes (outliers).

No geral, as concentrações dos estrogênios no esgoto tratado foram menores do que as do esgoto bruto. Padrão este que pode ser visto mais claramente em relação aos estrogênios naturais (estrona, estradiol e estriol). Já com relação ao estrogênio sintético (etinilestradiol), este decaimento é menos evidente. Tal resultado reflete a ação de processos de tratamento de esgotos na remoção destes compostos.

Observa-se ainda que a matriz esgoto tratado foi a que se mostrou com maior variabilidade para os diferentes estrogênios estudados. Tal constatação pode ser explicada pela adoção de diferentes tecnologias de tratamento de efluentes, bem como pela vazão de entrada e carga orgânica aplicada, o que, por consequência, acarreta em uma heterogeneidade das eficiências de degradação/remoção de estrogênios. 


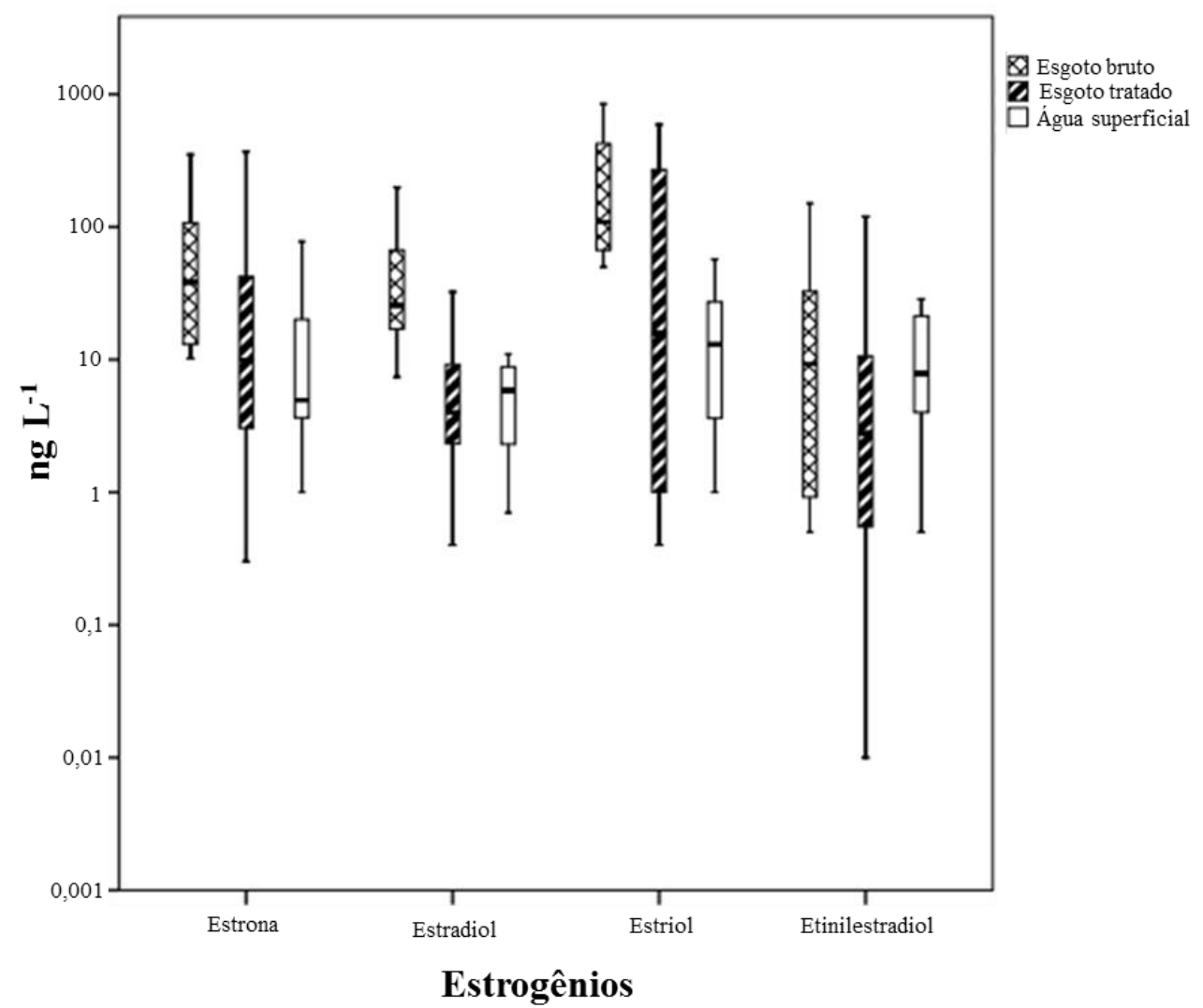

Figura 1. Variação das concentrações dos estrogênios estrona, estradiol, estriol e etinilestradiol no esgoto bruto, esgoto tratado e águas superficiais em diversos países (Drewes et al., 2005; Morteani et al., 2006; Chimchirian et al., 2007; Zorita et al., 2009; Duong et al., 2010; Zhang et al., 2011; Atkinson et al., 2012; Martin et al., 2012; Wang et al., 2012; Williams et al., 2012; Ye et al., 2012; Jin et al., 2013; Manickum e John, 2014; e Liu et al., 2015).

São ainda apresentados na sequência (Tabela 2) dados nacionais de concentrações de estrogênios encontrados nestas mesmas matrizes.

Embora tenham sido encontrados poucos artigos brasileiros sobre a ocorrência de estrogênios nas matrizes aquáticas, eles mostram que as concentrações destes compostos, com exceção do estriol (sobre o qual não foram encontrados estudos), foram bem superiores quando comparados aos dados relatados pelos estudos internacionais. Enquanto as concentrações destes estrogênios nos estudos internacionais não ultrapassaram a ordem de $1.000 \mathrm{ng} \mathrm{L}^{-1}$, no Brasil chegaram a $5.000 \mathrm{ng} \mathrm{L}^{-1} \mathrm{em}$ esgoto bruto, e até $4.000 \mathrm{ng} \mathrm{L}^{-1} \mathrm{em}$ águas superficiais (Tabela 2).

Outro ponto que merece atenção dentre os estudos de âmbito nacional, é ampla dispersão das concentrações, nas três matrizes estudadas. Os diferentes autores detectaram concentrações em ordens de grandeza bem distintas entre si, o que dificulta o estabelecimento de concentrações médias desses compostos para cada uma das matrizes. Tais variações podem ser explicadas por diversos fatores, como: condições locais, diferenças sociais e de saúde pública, e, ainda, diferenças entre as metodologias aplicadas para a condução de cada um dos estudos (Xu et al., 2012).

Mesmo com tal variabilidade entre os dados, é possível observar que ao analisar as concentrações obtidas por um mesmo autor, pode-se notar que há um significativo decaimento das concentrações desses estrogênios do esgoto bruto ao tratado (Pessoa et al., 2014; Queiroz et al., 2012; Froehner et al., 2011; Ternes et al., 1999), padrão este que confere com aquele 
observado nos estudos internacionais (Atkinson et al., 2012; Chimchirian et al., 2007; Duong et al., 2010; Manickum e John, 2014; Martin et al., 2012; Zhang et al., 2011; Zorita et al., 2009).

Tabela 2. Concentrações de estrogênios encontrados em esgoto bruto, esgoto tratado e águas superficiais do Brasil.

\begin{tabular}{|c|c|c|c|c|}
\hline Estrogênio & Matriz & Local & oncentração ng L ${ }^{-1}$ & Referência \\
\hline \multirow{7}{*}{ 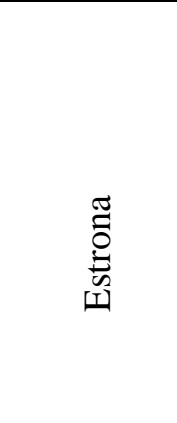 } & \multirow{3}{*}{$\begin{array}{l}\text { Esgoto } \\
\text { bruto }\end{array}$} & Curitiba & $870-1.380$ & Froehner et al. (2011) \\
\hline & & Fortaleza & 3.050 & Pessoa et al. (2014) \\
\hline & & Rio de Janeiro & 40 & Ternes et al. (1999) \\
\hline & \multirow{3}{*}{$\begin{array}{l}\text { Esgoto } \\
\text { tratado }\end{array}$} & Curitiba & $<\mathrm{LD}$ & Froehner et al. (2011) \\
\hline & & Fortaleza & 2.080 & Pessoa et al. (2014) \\
\hline & & Rio de Janeiro & 7 & Ternes et al. (1999) \\
\hline & $\begin{array}{l}\text { Água } \\
\text { superficial }\end{array}$ & $\begin{array}{l}\text { Atibaia, Campinas, } \\
\text { Paulínia e Americana }\end{array}$ & $<16$ & Montagner e Jardim (2011) \\
\hline \multirow{11}{*}{ 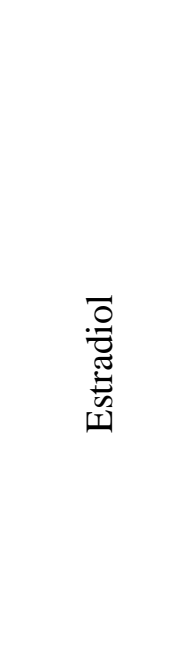 } & \multirow{4}{*}{$\begin{array}{l}\text { Esgoto } \\
\text { bruto }\end{array}$} & Belo Horizonte & $<9,3-31$ & Queiroz et al. (2012) \\
\hline & & Curitiba & $1.330-2.270$ & Froehner et al. (2011) \\
\hline & & Fortaleza & 776 & Pessoa et al. (2014) \\
\hline & & Rio de Janeiro & 21 & Ternes et al. (1999) \\
\hline & \multirow{4}{*}{$\begin{array}{l}\text { Esgoto } \\
\text { tratado }\end{array}$} & Belo Horizonte & $<9,3$ & Queiroz et al. (2012) \\
\hline & & Curitiba & $490-760$ & Froehner et al. (2011) \\
\hline & & Fortaleza & 397 & Pessoa et al. (2014) \\
\hline & & Rio de Janeiro & 1 & Ternes et al. (1999) \\
\hline & \multirow{3}{*}{$\begin{array}{l}\text { Água } \\
\text { superficial }\end{array}$} & $\begin{array}{l}\text { Atibaia, Campinas, } \\
\text { Paulínia e Americana }\end{array}$ & $<45-6.806$ & Montagner e Jardim (2011) \\
\hline & & Belo Horizonte & $<1-37$ & Moreira et al. (2009) \\
\hline & & $\begin{array}{l}\text { Ouro Preto, Itabirito } \\
\text { Rio Acima, Nova Lima }\end{array}$ & $<4-63$ & Moreira et al. (2011) \\
\hline \multirow{9}{*}{ 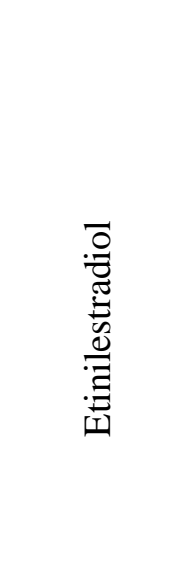 } & \multirow{3}{*}{$\begin{array}{l}\text { Esgoto } \\
\text { bruto }\end{array}$} & Belo Horizonte & $<12,4-41,3$ & Queiroz et al. (2012) \\
\hline & & Curitiba & $600-1.260$ & Froehner et al. (2011) \\
\hline & & Fortaleza & 3.180 & Pessoa et al. (2014) \\
\hline & \multirow{3}{*}{$\begin{array}{l}\text { Esgoto } \\
\text { tratado }\end{array}$} & Belo Horizonte & $<12,4$ & Queiroz et al. (2012) \\
\hline & & Curitiba & $<$ LD. -470 & Froehner et al. (2011) \\
\hline & & Fortaleza & 176 & Pessoa et al. (2014) \\
\hline & \multirow{3}{*}{$\begin{array}{l}\text { Água } \\
\text { superficial }\end{array}$} & $\begin{array}{l}\text { Atibaia, Campinas, } \\
\text { Paulínia e Americana }\end{array}$ & $<17-4.390$ & Montagner e Jardim (2011) \\
\hline & & Belo Horizonte & $<1-54$ & Moreira et al. (2009) \\
\hline & & $\begin{array}{l}\text { Ouro Preto, Itabirito, } \\
\text { Rio Acima, Nova Lima }\end{array}$ & $<5-64$ & Moreira et al. (2011) \\
\hline
\end{tabular}

Nota: LD - Limite de detecção.

Após o processo de tratamento de esgoto e seu lançamento nos corpos hídricos, os estrogênios são ainda submetidos a diferentes graus de atenuação natural que podem minimizar o seu impacto ambiental, como por exemplo, a diluição, a eventual adsorção em sólidos em suspensão e em sedimentos, a fotólise e a biodegradação aeróbia (Gómez et al., 2012; Pal et al., 2010). Mas há ainda que se considerar a possibilidade de ressuspensão destes compostos para coluna d'água, o que pode torná-los novamente mais biodisponíveis. 
Com relação às águas superficiais, no geral, a estrona é o estrogênio mais abundante e frequentemente detectado (Manickum e John, 2014; Jin et al., 2013; Wang et al., 2012; Duong et al., 2010; Kim et al., 2007). A maior ocorrência desse estrogênio nos corpos hídricos devese principalmente ao fato da estrona ser o principal produto da biodegradação do estradiol (Figura 2), durante os processos de tratamento de esgoto (Zorita et al., 2009). Segundo Sodré et al. (2007), dentre os estrogênios naturais, o estradiol é o mais potente, sendo estrogenicamente doze vezes mais ativo que a estrona. No que diz respeito ao etinilestradiol, por se tratar de um estrogênio sintético, sua ocorrência no meio hídrico pode ser diretamente relacionada ao consumo do mesmo, através de contraceptivos orais.

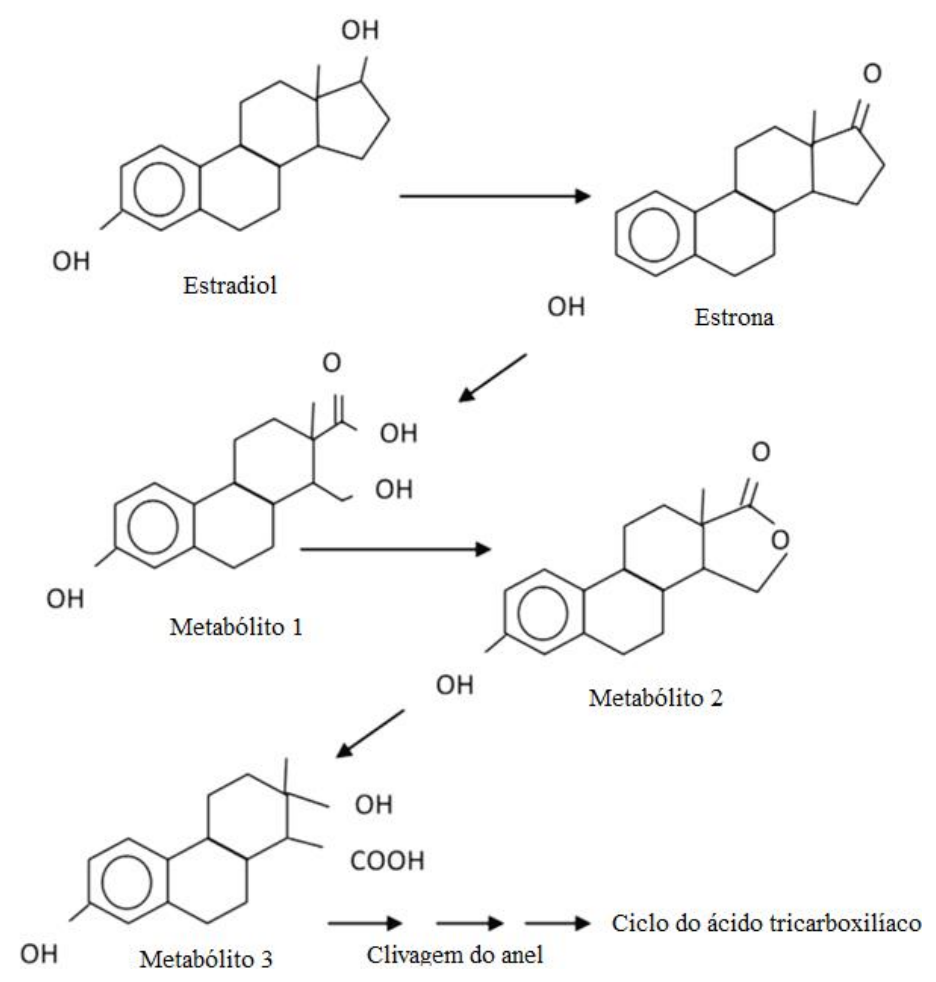

Figura 2. Via metabólica de degradação do estradiol por bactérias presentes no esgoto.

Fonte: Adaptado de Hamid e Eskicioglu (2012).

Caracterizar o comportamento dos estrogênios nas diferentes matrizes aquáticas apresenta-se como uma atividade complexa devido suas características físico-químicas, das propriedades do meio receptor e das inúmeras variáveis que atuam em conjunto no ambiente, como temperatura, turbidez, $\mathrm{pH}$, alcalinidade, oxigênio dissolvido, radiação, relações de sinergismo, atividade microbiana e concentração de outras substâncias. No entanto, o conhecimento desses compostos, seus níveis de ocorrência em matrizes ambientais, bem como a avaliação dos seus efeitos, configuram-se como atividades extremamente importantes (Bila e Dezotti, 2007).

\section{REMOÇÃO DE ESTROGÊNIOS POR PROCESSOS BIOLÓGICOS DE TRATAMENTO DE ESGOTO}

Os processos biológicos de tratamento são amplamente empregados no Brasil e no mundo. Dentre os processos de tratamento classificados como biológicos, o processo aeróbio por lodos ativados é o mais utilizado, seguido pelos sistemas anaeróbios, como o RAFA (Reator Anaeróbio de Fluxo Ascendente) - também conhecido como UASB "Upflow Anaerobic Sludge Blanket" - e as lagoas de estabilização. 
Atualmente, variantes destes diferentes processos foram e estão sendo desenvolvidos para melhorar a remoção de matéria orgânica e nutrientes (nitrogênio e fósforo). Por outro lado, os estudos envolvendo a remoção dos micropoluentes emergentes, como os estrogênios, partem da análise do potencial de aplicação destas variantes com tal finalidade.

Para comparar a eficiência da remoção de estrogênios, além das variações dos parâmetros de controle de processo de lodo ativado, como: tempo de retenção dos sólidos (TRS), tempo de detenção hidráulica (TDH), índice volumétrico do lodo, taxa de aeração, remoção de matéria orgânica (DBO/DQO), remoção de nitrogênio e fósforo; também são simuladas variações nos tipos de processos por lodos ativados, tais como: valos de oxidação, reator em batelada sequencial, processo anóxico/aeróbio, lodo ativado utilizando clarificador no final do processo, lodos ativados com posterior tratamento terciário e bactérias anaeróbias facultativas redutoras de ferro (Petrie et al., 2014; Shi et al., 2013; Kumar et al., 2011; Ivanov et., 2010; Hashimoto e Murakami, 2009).

Hashimoto e Murakami (2009) compararam as eficiências de remoção de estrogênios por lodos ativados convencional e por lodos ativados com valos de oxidação com variação do TRS. Esses autores observaram que o processo por lodos ativados convencional foi capaz de degradar praticamente todo o estradiol (mais de 95\%) à estrona com apenas cinco minutos de processo, em escala laboratorial; e a estrona, por sua vez, permaneceu posteriormente sorvida ao lodo após 24 horas de detenção hidráulica e pôde ser removida por sedimentação. Por outro lado, nos ensaios em que foi utilizado o lodo dos valos de oxidação, somente houve remoção pela sorção após uma hora de processo. No estágio anóxico do valo de oxidação, a remoção tanto da estrona quanto do estradiol foi menor (cerca de 20\%). Em ambos os processos, o etinilestradiol teve alta taxa de remoção (da ordem de $85 \%$ ), mas somente após oito horas de processo. Este estrogênio sintético não sofreu biodegradação, porém também foi removido pela sorção no lodo sedimentado. No geral, praticamente todas as variantes ensaiadas apresentaram maior eficiência na degradação/sorção dos estrogênios quando se adotou maiores TRS.

Shi et al. (2013) também relataram a degradação do estradiol à estrona em um reator independente da fase do processo (anóxica ou aeróbia). Porém, a transformação destes estrogênios na fase aeróbia foi mais intensificada. Pessoa et al. (2014) também encontraram maiores concentrações de estrona do que estradiol no efluente tratado por lodos ativados, devido à sua biodegradação aeróbia. O estudo de Shi et al. (2013) mostrou também que os estrogênios podem ser transferidos da fase líquida para o lodo, mostrando que os fenômenos de sorção podem ser os predominantes no processo de remoção desses compostos.

Kumar et al. (2011) obtiveram altas taxas de remoção dos estrogênios com valores acima de $80 \%$ em um processo por lodos ativados convencional adotando altos valores do TRS. Esses autores constataram que quanto menor a idade do lodo, menor é a taxa de remoção de estrogênios, o que corrobora com os resultados encontrados por Hashimoto e Murakami (2009). Entretanto, Petrie et al. (2014) obtiveram remoção de até 70\% de estrogênios com o TRS de três a dez dias. Isso mostra que ainda não existe um consenso quanto as variações dos parâmetros de controle dos processos de lodos ativados para uma melhor taxa de remoção e/ou degradação desses estrogênios.

No que diz respeito à eficiência de remoção de estrogênios em relação à operação em ambientes aeróbio, anóxico ou anaeróbio, estudos relataram que os sistemas aeróbios foram os mais eficazes. Ivanov et al. (2010) realizaram um estudo utilizando bactérias anaeróbias e facultativas com capacidade de redução de ferro para verificar a biodegradação dos estrogênios. Neste processo, tanto estrona quanto estriol foram reduzidos em $60 \%$ e $27 \%$, respectivamente. Com 15 dias de processo foi observada a remoção completa da estrona. Por outro lado, somente $9 \%$ do etinilestradiol foi removido, provavelmente pelo mecanismo de 
sorção. Este estudo indica que nos processos anaeróbios existem mecanismos de degradação diferentes do aeróbio, mas que a remoção pela sorção no lodo também pode ocorrer.

Com o propósito de avaliar o potencial de remoção e/ou degradação de estrogênios utilizando fontes alternativas de carbono orgânico, Racz et al. (2012) utilizaram dois reatores em bateladas sequenciais (RBS) em escala laboratorial utilizando esgoto sintético, onde em um foi adicionada peptona e, no outro, glicose como fonte de carbono. Os autores observaram uma maior eficiência de sorção pelo lodo no reator com peptona, o que foi explicado pela alta taxa de nitrificação. Este estudo sugere ainda que, sistemas aeróbios nitrificantes podem ter uma eficiência maior na remoção principalmente do estrogênio sintético etinilestradiol.

Além dos sistemas por lodos ativados e os anaeróbios, outros processos têm sido testados quanto a remoção de estrogênios como os sistemas de alagados (wetland) e lagoas (estabilização, maturação e suas diversas variantes). Pessoa et al. (2014) avaliaram a eficiência de remoção da estrona, estradiol, e etinilestradiol em quatro tipos de processos distintos em escala real: uma lagoa facultativa seguida por uma lagoa de maturação, uma lagoa facultativa, um sistema de lodos ativados e um reator UASB. As taxas de remoção desses estrogênios pelas lagoas foram abaixo de $70 \%$, enquanto que os processos de lodos ativados e reator UASB tiveram uma eficiência acima de $90 \%$.

Song et al. (2009) estudaram a remoção da estrona, estradiol e etinilestradiol em diferentes profundidades de um sistema de alagados (wetland) em escala piloto como pós-tratamento de lodos ativados. Os autores observaram uma maior eficiência (da ordem de 75\%), na remoção de estrogênios nas camadas mais superficiais do alagado, onde provavelmente prevalece o metabolismo aeróbio. Este estudo relatou que a biodegradação e a sorção foram os mecanismos predominantes nas zonas da rizosfera, onde prevalecem as raízes secundárias das plantas (raízes mais finas). Logo, micro-organismos presentes nas raízes das plantas podem estar atuando nos mecanismos de remoção e/ou degradação desses compostos.

Portanto, com base nos estudos consultados é possível observar que os processos aeróbios por lodos ativados parecem ser os mais promissores na remoção de estrogênios, sobretudo por serem os sistemas mais citados na literatura sobre este assunto. No entanto, para que seja consolidado este conhecimento é necessário associar alguns dos parâmetros operacionais adotados nesses sistemas como idade do lodo, TDH e taxa de aeração, por exemplo, com a eficiência de remoção e/ou degradação desses compostos. Além disso, faz-se pertinente analisar como a adoção de processos anóxicos e/ou anaeróbios podem influenciar nas condições metabólicas, e por consequência na remoção e/ou degradação destes compostos.

Um ponto ainda pouco estudado e que merece atenção é o fato de, ao se desencadear o processo de biodegradação dos estrogênios, haver a produção de metabólitos. Desta forma, é importante que mais estudos investiguem tais mecanismos de biodegradação ao longo dos sistemas e, sobretudo, identifiquem quais subprodutos podem ser formados e seus respectivos potenciais de desregulação endócrina.

\section{CONCLUSÃO}

Os estrogênios apresentam-se como importantes contaminantes emergentes que podem afetar a saúde ambiental e humana. Estes têm no lançamento de esgotos domésticos sua principal via de introdução no meio ambiente. Estudos realizados em diversos países relataram concentrações significativas desses compostos em esgoto bruto e tratado, bem como nas águas superficiais que recebem esgoto doméstico. Apesar de existirem poucos artigos sobre este assunto, os níveis desses contaminantes no Brasil são maiores do que os encontrados nos demais países. Os sistemas de tratamento de esgotos podem representar uma importante barreira neste processo de contaminação do meio hídrico. Entretanto, no Brasil, em decorrência dos baixos 
índices de coleta e tratamento de esgotos, ainda se observa que boa parte dos estrogênios excretados acaba tendo como destino final os corpos hídricos (SNIS, 2013).

Os processos aeróbios, sobretudo os por lodos ativados, mostraram melhor desempenho na biodegradação desses compostos. A sorção pelo lodo biológico parece ser o principal mecanismo na remoção de estrogênios; no entanto, ainda é necessário conhecer melhor os mecanismos de sua biodegradação. Há uma vasta gama de possibilidades tecnológicas que podem remover e/ou degradar esses compostos. Porém ainda são necessários mais estudos sobre a utilização destas tecnologias de tratamento no Brasil. Esta área de conhecimento merece maior atenção, uma vez que as ETEs podem atuar como importantes barreiras, tanto à introdução de estrogênios, quanto de outros micropoluentes emergentes.

\section{AGRADECIMENTOS}

Os autores agradecem ao estatístico Felipe Pinto pelo apoio na análise e apresentação gráfica dos dados, à Capes pela concessão de bolsa de doutorado, à Faperj (Processos E26/110.102/2014 e E-26/201.479/2014) e ao CNPq (Processo 45533/2014-7) pelo apoio a pesquisa.

\section{REFERÊNCIAS}

AQUINO, S. F.; BRANDT, E. M. F.; CHERNICHARO, C. A. L. Remoção de fármacos e desreguladores endócrinos em estações de tratamento de esgoto: revisão da literatura. Engenharia Sanitária e Ambiental, v.18, n. 3, p. 187-204, 2013. http://dx.doi.org/10.1590/S1413-41522013000300002

ATKINSON, S. K.; MARLATT, V. L.; KIMPE, L. E. et al. The occurrence of steroidal estrogens in south-eastern Ontario wastewater treatment plants. Science of the Total Environment, v. 430, p. 119-125, 2012.

http://dx.doi.org/10.1016/j.scitotenv.2012.04.069.

BARCELÓ, D.; PETROVIC, M. Emerging contaminants from industrial and municipal waste: occurrence, analysis and effects. Berlin: Springer; c2008. 206p.

BERGMAN, A.; HEINDEL, J. J.; JOBLING, S. et al. State of the science of endocrine disrupting chemicals. Genebra: UNEP; WHO, c2012. 180p.

BILA, D. M.; DEZOTTI, M. Desreguladores endócrinos no meio ambiente: efeitos e consequências. Química Nova, v. 30, n. 3, p. 651-666, 2007. http://dx.doi.org/10.1590/S0100-40422007000300027.

BRANDT, E. M. F; QUEIROZ, F. B.; AFONSO, R. J. C. F. et al. Behaviour of pharmaceuticals and endocrine disrupting chemicals in simplified sewage treatment systems. Journal of

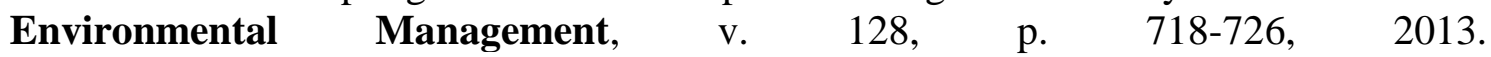
http://dx.doi.org/10.1016/j.jenvman.2013.06.003.

CANO, A.; ROURA, A. C.; CORTIT, L. I. Farmacología de los anticonceptivos hormonales orales. In: BUIL, C. (Ed.). Manual de anticoncepcíon hormonal oral. Zaragoza: Sociedad Española de Contracepción, 1997. p. 75-99.

CETESB - COMPANHIA DE TECNOLOGIA DE SANEAMENTO AMBIENTAL. Relatório de estabelecimento de valores orientadores para solos e águas subterrâneas no Estado de São Paulo. São Paulo, 2001. 
CHIMCHIRIAN, R. F.; SURI, R. P. S.; FU, H. Free synthetic and natural estrogen hormones in influent and effluent of three municipal wastewater treatment plants. Water $\begin{array}{llllllll}\text { Environment } & \text { Research, } & \text { v. 79, } & \text { n. 9, }\end{array}$ http://www.jstor.org/stable/25053673.

COMBALBERT, S.; HERNANDEZ-RAQUET, G. Occurrence, fate, and biodegradation of estrogens in sewage and manure. Applied Microbiology and Biotechnology, v. 86, n. 6, p. 1671-1692, 2010. http://dx.doi.org/10.1007/s00253-010-2547-x

CUNHA, D. L.; SILVA, S. M. C.; BILA, D. M. et al. Regulamentação do estrogênio sintético $17 \alpha$-etinilestradiol em matrizes aquáticas na Europa, Estados Unidos e Brasil. Cadernos de Saúde Pública, v. 32, n. 3, p. 1-13, 2016. http://dx.doi.org/10.1590/0102311 X00056715.

D'ASCENZO, G.; DI CORCIA, A.; GENTILI, A. et al. Fate of natural estrogen conjugates in municipal sewage transport and treatment facilities. Science of the Total Environment, v. 302, n. 1-3, p. 199-209, 2003. http://dx.doi.org/10.1016/S0048-9697(02)00342-X

DREWES, J. E.; HEMMING, J.; LADENBURGER, S. et al. An assessment of endocrine disrupting activity changes in water reclamation systems through the use of bioassays and chemical measurements. Water Environment Research, v. 77, n. 1, p. 12-23, 2005. http://dx.doi.org/10.2175/106143005X41573.

DUONG, C. N.; RA, J. S.; CHO J. et al. Estrogenic chemicals and estrogenicity in river waters of South Korea and seven Asian countries. Chemosphere, v. 78, p. 286-293, 2010. http://dx.doi.org/10.1016/j.chemosphere.2009.10.048.

FENT, K.; WESTON, A. A.; CAMINADA, D. Ecotoxicology of human pharmaceuticals. Aquatic toxicology, v. 76, n. 2, p. 122-159, 2006. http://dx.doi.org/10.1016/j.aquatox.2005.09.009

FROEHNER, S.; PICCIONI, W.; MACHADO, K. S.; AISSE, M. M. Removal capacity of caffeine, hormones, and bisphenol by aerobic and anaerobic sewage treatment. Water, Air \& Soil Pollution, v. 216, n. 1, p. 463-471, 2011. http://dx.doi.org/10.1007/s11270010-0545-3.

GARMSHAUSEN, J.; KLOAS, W.; HOFFMANN, F. 17 $\alpha$-ethinylestradiol can disrupt hemoglobin catabolism in amphibians. Comparative Biochemistry and Physiology Part C: Toxicology \& Pharmacology, v. 171, p. 34-40, 2015. http://dx.doi.org/10.1016/j.cbpc.2015.03.004.

GILBERT, N. Drug-pollution law all washed up. Nature, v. 491, n. 7425, p. 503-504, 2012. http://dx.doi.org/10.1038/491503a.

GIUSTI, A.; LAGADIC, L.; BARSI, A. et al. Investigating apical adverse effects of four endocrine active substances in the freshwater gastropod Lymnaea stagnalis. Science of $\begin{array}{llllll}\text { the Total Environment, } & \text { v. 493, p. 147-155, }\end{array}$ http://dx.doi.org/10.1016/j.scitotenv.2014.05.130.

GÓMEZ, M. J.; HERRERA, S.; SOLÉ, D. et al. Spatio-temporal evaluation of organic contaminants and their transformation products along a river basin affected by urban, agricultural and industrial pollution. Science of The Total Environment, v. 420, p.134145, 2012. http://dx.doi.org/10.1016/j.scitotenv.2012.01.029. 
HAMID, H; ESKICIOGLU, C. Fate of estrogenic hormones in wastewater and sludge treatment: A review of properties and analytical detection techniques in sludge matrix. $\begin{array}{lllllll}\text { Water Research, } & \text { v. 46, n. 18, p. 5813-33, }\end{array}$ http://dx.doi.org/10.1016/j.watres.2012.08.002.

HASHIMOTO, T.; MURAKAMI, T. Removal and degradation characteristics of natural and synthetic estrogens by activated sludge in batch experiments. Water Research, v. 43, n. 3, p. 573-582, 2009. http://dx.doi.org/10.1016/j.watres.2008.10.051.

IARC - INTERNATIONAL AGENCY FOR RESEARCH ON CANCER. IARC monographs on the evaluation of carcinogenic risks to humans. Lyon, 2007.

IVANOV, V.; LIM, J.; STABNIKOVA, O.; GIN, K. Biodegradation of estrogens by facultative anaerobic iron-reducing bacteria. Process Biochemistry, v. 45, n. 2, 284-287, 2010. http://dx.doi.org/10.1016/j.procbio.2009.09.017.

JIN, S.; YANG, F.; XU, Y.; DAI, H.; LIU, W. Risk assessment of xenoestrogens in a typical domestic sewage-holding lake in China. Chemosphere, v. 93, n. 6, p. 892-8, 2013. http://dx.doi.org/10.1016/j.chemosphere.2013.05.037.

KIM, S. D.; CHO, J.; KIM, In S. et al. Occurrence and removal of pharmaceuticals and endocrine disruptors in South Korean surface, drinking, and waste waters. Water research, v. 41, n. 5, p. 1013-1021, 2007. http://dx.doi.org/10.1016/j.watres.2006.06.034

KUMAR, V.; NAKADA, N.; YASOJIMA, M. et al. The arrival and discharge of conjugated estrogens from a range of different sewage treatment plants in the UK. Chemosphere, v. 82, n. 8, p. 1124-1128, 2011. http://dx.doi.org/10.1016/j.chemosphere.2010.11.040.

LIU, J.; LU, G.; XIE, Z. et al. Occurrence, bioaccumulation and risk assessment of lipophilic pharmaceutically active compounds in the downstream rivers of sewage treatment plants. Science of the Total Environment, v. 511, p. 54-62, 2015. http://dx.doi.org/10.1016/j.scitotenv.2014.12.033.

LUNA, T. O.; PLAUTZ, S. C.; SALICE, C. J. Chronic effects of 17 $\alpha$-ethinylestradiol, fluoxetine, and the mixture on individual and population-level end points in Daphnia magna. Archives of Environmental Contamination and Toxicology, v. 68, n. 4, p. 603-611, 2015. http://dx.doi.org/10.1007/s00244-014-0119-2.

MANICKUM, T.; JOHN, W. Occurrence, fate and environmental risk assessment of endocrine disrupting compounds at the wastewater treatment works in Pietermaritzburg (South Africa). Science of the Total Environment, v. 468-469, p. 584-597, 2014. http://dx.doi.org/10.1016/j.scitotenv.2013.08.041.

MARTIN, J.; CAMACHO-MUÑOZ, D.; SANTOS, J. L. et al. Occurrence of pharmaceutical compounds in wastewater and sludge from wastewater treatment plants: Removal and ecotoxicological impact of wastewater discharges and sludge disposal. Journal of Hazardous Materials, v. 239-240, p. 40-47, 2012. http://dx.doi.org/10.1016/j.jhazmat.2012.04.068.

MONTAGNER, C. C.; JARDIM, W. F. Spatial and seasonal variations of pharmaceuticals and endocrine disruptors in the Atibaia river, São Paulo State (Brazil). Journal of the Brazilian Chemical Society, v. 22, n. 8, p. 1452-1462, 2011. http://dx.doi.org/10.1590/S0103-50532011000800008. 
MOREIRA, D. S.; AQUINO, S. F.; AFONSO, R. J. C. F. et al. Occurrence of endocrine disrupting compounds in water sources of Belo Horizonte Metropolitan Area, Brazil.

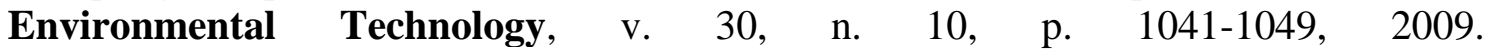
http://dx.doi.org/10.1080/09593330903052830.

MOREIRA, M. A.; AQUINO, S. F.; COUTRIM, M. X. et al. Determination of endocrinedisrupting compounds in waters from Rio das Velhas, Brazil, by liquid chromatography/high resolution mass spectrometry (ESILC-IT-TOF/MS). Environmental Technology, v. 32, n. 11-12, p. 1409-1417, 2011. http://dx.doi.org/10.1080/09593330.2010.537829.

MORTEANI, G.; MÖLLER, P.; FUGANTI, A.; PACES, T. Input and fate of anthropogenic estrogens and gadolinium in surface water and sewage plants in the hydrological basin of Prague (Czech Republic). Environmental Geochemistry and Health, v. 28, n. 3, p. 25764, 2006. http://dx.doi.org/10.1007/s10653-006-9040-6.

PAL, A.; GIN, K. Y. H.; LIN, A. Y. C.; REINHARD, M. Impacts of emerging organic contaminants on freshwater resources: review of recent occurrences, sources, fate and effects. Science of The Total Environment, v. 408, n. 24, p. 6062-6069, 2010. http://dx.doi.org/10.1016/j.scitotenv.2010.09.026.

PESSOA, G. P.; SOUZA, N. C.; VIDAL, C. B. et al. Occurrence and removal of estrogens in Brazilian wastewater treatment plants. Science of the Total Environment, v. 490, 288295, 2014. http://dx.doi.org/10.1016/j.scitotenv.2014.05.008.

PETRIE, B.; McADAM, E.; HASSARD, F. et al. Diagnostic investigation of steroid estrogen removal by activated sludge at varying solids retention time. Chemosphere, v. 113, p. 101-108, 2014. http://dx.doi.org/10.1016/j.chemosphere.2014.04.051.

QUEIROZ, F. B.; BRANDT, E. M. F.; AQUINO, S. F. et al. Occurrence of pharmaceuticals and endocrine disruptors in raw sewage and their behavior in UASB reactors operated at different hydraulic retention times. Water Science \& Technology, v. 6612, p. 25622569, 2012. http://dx.doi.org/10.2166/wst.2012.482

RACZ, L.; MULLER, J.; GOEL, R. Fate of selected estrogens in two laboratory scale sequencing batch reactors fed with different organic carbon sources under varying solids retention times. Bioresource Technology, v. 110, p. 35-42, 2012. http://dx.doi.org/10.1016/j.biortech.2012.01.059.

SHI, J.; CHEN, Q.; LIU, X. et al. Sludge/water partition and biochemical transformation of estrone and $17 \beta$-estradiol in a pilot-scale step-feed anoxic/oxic wastewater treatment system. Biochemical Engineering Journal, v. 74, p. 107-114, 2013. http://dx.doi.org/10.1016/j.bej.2013.03.001.

SILVA, P.; ROCHA, M. J.; CRUZEIRO, C. et al. Testing the effects of ethinylestradiol and of an environmentally relevant mixture of xenoestrogens as found in the Douro River (Portugal) on the maturation of fish gonads--a stereological study using the zebrafish (Danio rerio) as model. Aquatic Toxicology, v. 124-125, p. 1-10, 2012. http://dx.doi.org/10.1016/j.aquatox.2012.07.002.

SNIS - SISTEMA NACIONAL DE INFORMAÇÃO EM SANEAMENTO (Brasil). Série Histórica. 2013. Disponível em: http://app.cidades.gov.br/serieHistorica/manuais/ae/ diagnosticos/DiagAE_2013.pdf. Acesso em: 2016. 
SODRÉ, F. F. M.; MONTAGNER, C. C.; LOCATELLI, M. A. F.; JARDIM, W. F. Ocorrência de interferentes endócrinos e produtos farmacêuticos em águas superficiais da Região de Campinas (SP, Brasil). Journal of the Brazilian Society of Ecotoxicology, v. 2, n. 2, p. 187-196, 2007.

SONG, H.; NAKANO, K.; TANIGUCHI, T. et al. Estrogen removal from treated municipal effluent in small-scale constructed wetland with different depth. Bioresource Technology, v. 100, n. 12, p. 2945-2951, 2009. http://dx.doi.org/10.1016/j.biortech.2009.

TERNES, T. A.; STUMPF, M.; MUELLER, J. et al. Behavior and occurrence of estrogens in municipal sewage treatment plants - 1: Investigations in German, Canada and Brazil. Science of the Total Environment, v. 225, n. 1-2, p. 81-90, 1999. http://dx.doi.org/10.1016/S0048-9697(98)00334-9.

WILLIAMS, R. J.; CHURCHLEY, J. H. et al. Comparing predicted against measured steroid estrogen concentrations and the associated risk in two United Kingdom river catchments. Environmental Toxicology and Chemistry, v. 31, n. 4, p. 892-898, 2012. http://dx.doi.org/10.1002/etc.1756.

WANG, G.; MA, P.; ZHANG, Q. et al. Endocrine disrupting chemicals in New Orleans surface waters and Mississippi Sound sediments. Journal of Environmental Monitoring, v. 14, n. 5, p. 1353-1364, 2012. http://dx.doi.org/10.1039/c2em30095h.

XU, N.; XU, Y.; XU, S. et al. Removal of estrogens in municipal wastewater treatment plants: A Chinese perspective. Environmental Pollution, v. 165, p. 215-224, 2012. http://dx.doi.org/10.1016/j.envpol.2011.12.025

YE, X.; GUO, X.; CUI, X. et al. Occurrence and removal of endocrine-disrupting chemicals in wastewater treatment plants in the Three Gorges Reservoir area, Chongqing, China. Journal of Environmental Monitoring, v. 14, n. 8, p. 2204-11, 2012. http://dx.doi.org/10.1039/c2em30258f.

ZHANG, Z.; FENG, Y.; GAO, P. et al. Occurrence and removal efficiencies of eight EDCs and estrogenicity in a STP. Journal of Environmental Monitoring, v. 13, n. 5 p. 1366-1373, 2011. http://dx.doi.org/10.1039/c0em00597e.

ZORITA, S.; MÅRTENSSON, L.; MATHIASSON, L. Occurrence and removal of pharmaceuticals in a municipal sewage treatment system in the south of Sweden. Science of the Total Environment, v. 407, n. 8, p. 2760-2770, 2009. http://dx.doi.org/10.1016/j.scitotenv.2008. 